\title{
Wave motion between two fluid-filled boreholes in an elastic medium
}

\author{
António Tadeu* , Luís Godinho, Paulo Santos \\ Faculty of Science and Technology, Department of Civil Engineering, University of Coimbra-Polo II, Pinhal de Marrocos, 3030-290 Coimbra, Portugal
}

Received 25 April 2001; revised 1 August 2001; accepted 8 August 2001

\begin{abstract}
The boundary element method (BEM) is used to fully simulate the propagation of waves between two fluid-filled boreholes. The sources are placed in one of the boreholes while the receivers are placed in the other. This model is frequently used in cross-hole seismic prospecting techniques to assess the characteristics of the elastic medium between the two boreholes. This work studies the dependence of the wave propagation patterns on the distance between the source and the receiver, their location and orientation relative to the axis of a circular borehole and type of elastic formation (fast and slow formations). In addition, this BEM model is used to compute the influence of the deformed boreholes whose cross-section is not circular. Both the spectra responses and the time-domain responses are computed to elucidate the main physical features of the problem solved. (C) 2002 Elsevier Science Ltd. All rights reserved.
\end{abstract}

Keywords: Cross-hole seismic prospecting techniques; Wave propagation; Boundary element method; Two-and-a-half-dimensional problem

\section{Introduction}

A number of geophysical and seismic prospecting techniques involve measuring the pressure inside a fluid-filled borehole, generated by a source on the surface or in another borehole [1-5].The cross-hole seismic reflection method, in particular, has undergone significant development in terms of acquisition, processing, and imaging capability. Findlay et al. [6] were among the first to publish stacked, depthmigrated sections from cross-hole surveys with multiple source and receiver positions. Stacked sections formed by reflection point mapping of real cross-hole data sets have also been produced by Goulty et al. [7], Becquey et al. [8], Lazaratos et al. [9] and Khalil et al. [10]. It has been recognized, however, that wavefield separation for cross-hole data is problematical $[11,12]$.

Different numerical methods have been developed for approaching the solution of the wave propagation across fluid-filled boreholes more realistically, looking for a better understanding of the data recorded at the receivers.

Direct modeling methods have been used to solve the differential equations governing the acoustic wave motion in the borehole fluid and in the solid formation simultaneously. For example, Lee and Balch [13] used the frequency axial wavenumber integration technique. By

\footnotetext{
* Corresponding author. Tel.: +351-239-797-201; fax: +351-239-797190.

E-mail address: tadeu@dec.uc.pt (A. Tadeu).
}

considering a low-frequency approximation, they only retained the relevant tube-wave terms and obtained a farfield asymptotic representation for the space-time domain displacement in a fast formation. Lee et al. [14] generalized the technique of Lee and Balch [13], incorporating the secondary radiation from totally reflected tube waves at the bottom of a borehole. Lee [15] investigated non-axisymmetric solutions for the acoustic wave motion in fast formations, while Winbow [16] modeled the influence of different source types and the presence of a borehole casing.

The effects of borehole irregularities on the Stoneley wave propagation have been addressed by Stephen et al. [17], using a finite difference scheme. Bouchon and Schmitt [18] used a boundary integral equation approach, combining the discrete wavenumber formulation, and concluded that when the change in the borehole diameter was smooth the Stoneley wave propagation was not affected, but a significant amount of reflection was computed in the case of a sharp variation in the diameter. Randall [19] studied monopole and dipole acoustic logs using a staggered grid in a 2-D cylindrical coordinate. He also studied the multipole acoustic $\log$ in a non-axisymmetric borehole using the staggered grid finite difference method. In this case the model is assumed to be invariant in the axial $z$ direction, which allows the spatial Fourier transform (2.5-D finite difference method). Leslie and Randall [20] extended their 2.5-D finite difference method to model acoustic wave propagation in a borehole penetrating a general anisotropic formation. A true 3-D finite difference method was applied to the acoustic 
logging of boreholes by Yoon and McMechan [21], using a second order scheme and assuming an isotropic formation. Later, Cheng et al. [22] used massive parallel computing to develop a 3-D finite difference time domain method for borehole wave propagation in an anisotropic formation, using a fourth order discretization in space. Most applications of the finite difference method, however, are still reserved for the 2-D geometries, because of the computational costs involved.

Finite difference methods have also been implemented by Track and Daube [23], who addressed the cross-borehole wave propagation problem, and by Cheng et al. [24], who concentrated on the coupling of the acoustic wave motion in the borehole fluid to conical $\mathrm{P}$ and $\mathrm{S}$ waves in a slow formation.

To allow practical field applications, hybrid modeling techniques have been proposed, based on the prior knowledge of the behavior of the dynamic system being analyzed. White and Sengbush [25] applied a hybrid modeling technique by recognizing that, at low frequencies, the tube wave dominates the acoustic wave motion in the borehole fluid. They subsequently introduced the motion of moving sources to model the acoustic tube-wave radiation into the formation, using Heelan's [26] far field expressions for the acoustic radiation caused by a transient pressure applied on a circular cylinder of finite extent.

Ben-Menahem and Kostek [27] employed a fixed system of equivalent seismic sources to simulate the influence of the borehole on the acoustic wave motion, by matching its far-field radiation characteristics with the far-field radiation characteristics of a fluid-filled borehole in a fast formation, as obtained by Lee and Balch [13]. Kurkjian and co-workers $[28,29]$ extended this approach by replacing the wave field radiated by the source borehole with a moving system of effective seismic sources, to account for the conical S-wave radiation into slow formations. Furthermore, they employed a frequency lateral wavenumber integration code to determine the seismic wave-field quantities in the formation. Gibson [30] also employed a moving system of effective seismic sources and calculated the wave-field quantities in slow and fast formation using ray asymptotics.

Hoop et al. [31] describe a hybrid method by which the transfer of transient tube-wave signals in cross-borehole experiments are calculated, assuming that the travel times of the elastic waves in the formation over distances of the order of a borehole diameter may be neglected. This means that the wavelengths involved are considerably longer than a borehole diameter, for both cased and uncased boreholes. Analysis is performed in the complex-frequency domain and uses the knowledge that, in the low-frequency domain, the axisymmetric wave motion in the borehole fluid is dominated by tube waves. Then the acoustic pressure on the axis of the receiving borehole is evaluated with the aid of the fluid/solid acoustic reciprocity theorem.

Kurkjian et al. [32] proposed a numerical technique for modeling downhole seismic data in crosswell configura- tions, dividing the problem into three distinct parts: generation of the source well representation, where the tube waves in the source well are taken into account; transmission from source well, using a preexisting code; computation of the hydrophone measurements by applying White's quasistatic approximation. They discretized the boreholes into small elements (10 points per tube wave wave-length). Later, Peng et al. [33] made use of both the borehole coupling theory and the global matrix formulation for computing synthetic seismograms in a layered medium. The global matrix formulation is used to calculate the stress field at the borehole location. Borehole coupling theory is then employed to obtain the pressure in the borehole fluid. No discretization along the borehole is required in this model, and the method gives results for open, cased and partially filled boreholes.

Tang and Cheng [34] addressed the computational speed problem by studying the Stoneley wave interactions caused by formation changes. They used a simple 1-D approach, assuming that the Stoneley wave propagates along the borehole with no geometric spreading, because it is a guided wave. Tezuka et al. [35] formulated a method for modeling the low-frequency Stoneley wave propagation, that arises in an irregular borehole as a result of the variation in the spatial diameter of the borehole along it axis, and formation property changes. The essential features of the low frequency Stoneley waves are retained with a single 1-D model. A mass-balance boundary condition and a propagator matrix are used to express Stoneley wave interactions with the borehole irregularities.

A large number of techniques have been based on approximations to exact solutions. The Boundary Element Method (BEM) is probably the best tool for analyzing wave propagation in the vicinity of borehole geometry, because it automatically satisfies the far field conditions. Bouchon [36] used the method in an infinite open borehole in layered isotropic media. Dong et al. [37] broadened the scope of Bouchon's work by incorporating transversely isotropic layers and by including the effect of casing and cement in the formation. Their work used an indirect BEM to model source radiation from open and cased boreholes in layered transversely isotropic media.

The effect of the irregular boreholes on the response has also been studied by Randall [38] and Tadeu et al. [39]. These effects result from the mechanical action of the drill string in vertically deviated wells, rock failure adjacent to a drilled borehole, plastic deformation and washing out of the borehole in soft or poorly consolidated rocks, as reported by Bell et al. [40] and by Zheng et al. [41].

The present work employs the BEM to simulate a full crosshole seismic prospecting technique, using two fluidfilled boreholes. Both boreholes are modeled with boundary elements and the computed responses fully represent the phenomena involved, without using any simplification. The source is placed in one of the boreholes, while the receivers are placed in the second borehole. The distance 
between boreholes, the presence of off-center sources and receivers, and the type of formation are analyzed.

The cylindrical geometry of this problem (Fig. 1) enables the solution at each frequency to be expressed as an integration of responses for a continuous variation of wavenumber, $k_{z}$, (with $z$ being the borehole axis). The discrete form of this integration is achieved by considering an infinite number of virtual point sources equally spaced along the $z$ direction. The proper definition of distances between these sources and the use of complex frequencies avoids spatial contamination and minimizes the influence of neighboring fictitious sources. A BEM formulation using compact expressions of Green's functions for the elastic and fluid media is used to solve this problem.

First, the formulation of the BEM model in the frequency domain is briefly described. Then, the characteristics of the dispersion of waves in a fluid-filled circular borehole are addressed. The main part of this work is devoted to numerical simulations. First, the effect on the response of the position of the receivers and sources, the distance between boreholes, and the type of elastic formation are analyzed. Then, the effect of the presence of irregular boreholes is examined. All the computations are achieved for a wide range of frequencies and wavenumbers, which are then used to obtain time series by means of (fast) inverse Fourier transforms into space-time.

\section{Problem formulation}

Consider an elastic medium that allows a shear wave velocity of $\beta$ and a compressional wave velocity of $\alpha$ with density $\rho$. Two boreholes are driven along the $z$ direction and filled with fluid to permit a compressional wave velocity $\alpha_{f}$ with density $\rho_{f}$. A dilatational point source is placed in one of the boreholes at position $\left(x_{0}, y_{0}, z_{0}\right)$, oscillating with a frequency $\omega$. The incident field can be expressed by the dilatational potential $\phi$

$\phi_{\text {inc }}=\frac{A \mathrm{e}^{i \frac{\omega}{\alpha_{f}}\left(\alpha t-\sqrt{\left(x-x_{0}\right)^{2}+\left(y-y_{0}\right)^{2}+\left(z-z_{0}\right)^{2}}\right)}}{\sqrt{\left(x-x_{0}\right)^{2}+\left(y-y_{0}\right)^{2}+\left(z-z_{0}\right)^{2}}}$

in which $A$ is the wave amplitude and $i=\sqrt{-1}$.

This problem can be solved as a summation of twodimensional problems, for varying effective wavenumbers [42],

$k_{\alpha f}=\sqrt{\frac{\omega^{2}}{\alpha_{f}^{2}}-k_{z}^{2}}, \quad \operatorname{Im} k_{\alpha f}<0$

where $k_{z}$ is the axial wavenumber after Fourier transformation of the problem in the $z$ direction. The incident field in this frequency wavenumber domain is given by

$\hat{\phi}_{\text {inc }}\left(\omega, x, y, k_{z}\right)=(-i A / 2) H_{0}^{(2)}\left(k_{\alpha f} \sqrt{\left(x-x_{0}\right)^{2}+\left(y-y_{0}\right)^{2}}\right)$

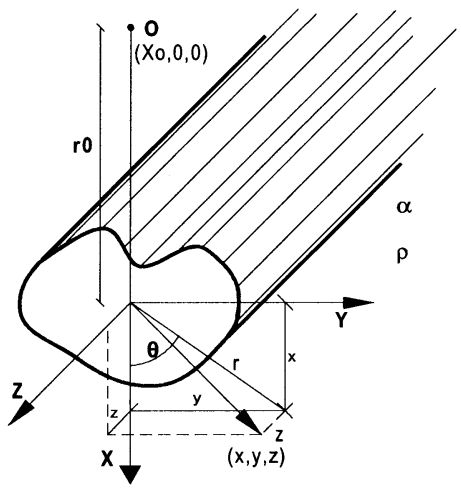

Fig. 1. Geometry of the problem.

in which the $H_{n}^{(2)}(\ldots)$ are second Hankel functions of order $n$.

The BEM gives the solution by discretizing only the boundary of the boreholes. The BEM equations that are applied to this problem are well known [43-45]. The system of equations required for the solution is arranged so as to impose the continuity of the normal displacements and normal stresses and null shear stresses along the boundary of the fluid-filled boreholes. This system of equations requires the evaluation of the following integrals along the appropriately discretized boundary of the borehole

$H_{i j}^{(s) k l}=\int_{C_{l}} H_{i j}^{(s)}\left(x_{k}, x_{l}, n_{l}\right) \mathrm{d} C_{l} \quad(i, j=1,2,3)$

$H_{f 1}^{(f) k l}=\int_{C_{l}} H_{f 1}^{(f)}\left(x_{k}, x_{l}, n_{l}\right) \mathrm{d} C_{l}$

$G_{i j}^{(s) k l}=\int_{C_{l}} G_{i j}^{(s)}\left(x_{k}, x_{l}\right) \mathrm{d} C_{l} \quad(i=1,2,3 ; j=1)$

$G_{f 1}^{(f) k l}=\int_{C_{l}} G_{f 1}^{(f)}\left(x_{k}, x_{l}\right) \mathrm{d} C_{l}$

in which $H_{i j}^{(s)}\left(x_{k}, x_{l}, n_{l}\right)$ and $G_{i j}^{(s)}\left(x_{k}, x_{l}\right)$ are, respectively, the Green's tensor for traction and displacement components in the elastic medium, at point $x_{l}$ in direction $j$ caused by a concentrated load acting at the source point $x_{k}$ in direction $i$; $H_{f 1}^{(f)}\left(x_{k}, x_{l}, n_{l}\right)$ are the components of the Green's tensor for pressure in the fluid medium, at point $x_{l}$ caused by a pressure load acting at the source point $x_{k} ; G_{f 1}^{(f)}\left(x_{k}, x_{l}\right)$ are the components of the Green's tensor for displacement in the fluid medium, at point $x_{l}$ in the normal direction, caused by a pressure load acting at the source point $x_{k} ; n_{l}$ is the unit outward normal for the $l$ th boundary segment $\mathrm{C}_{l}$; the subscripts $i, j=1,2,3$ denote the normal, tangential and $z$ directions, respectively. These equations are conveniently transformed from the $x, y, z$ Cartesian coordinate system by means of standard vector transformation operators. The required two-and-a-half dimensional fundamental solution (Green's functions) and stress functions in Cartesian 
Table 1

Mechanical properties of the two formations

\begin{tabular}{ll}
\hline Fast formation & Slow formation \\
\hline$\alpha=4208 \mathrm{~m} / \mathrm{s}$ & $\alpha=2630 \mathrm{~m} / \mathrm{s}$ \\
$\beta=2656 \mathrm{~m} / \mathrm{s}$ & $\beta=1416 \mathrm{~m} / \mathrm{s}$ \\
$\rho=2140 \mathrm{~kg} / \mathrm{m}^{3}$ & $\rho=2250 \mathrm{~kg} / \mathrm{m}^{3}$ \\
$\alpha_{f}=1500 \mathrm{~m} / \mathrm{s}$ & $\alpha_{f}=1500 \mathrm{~m} / \mathrm{s}$ \\
$\rho_{f}=1000 \mathrm{~kg} / \mathrm{m}^{3}$ & $\rho_{f}=1000 \mathrm{~kg} / \mathrm{m}^{3}$ \\
\hline
\end{tabular}

co-ordinates, for the elastic and fluid media, are listed in Appendix A.

The required integrations in Eq. (4) are performed analytically for the loaded element [46,47], and using a Gaussian quadrature scheme when the element to be integrated is not the loaded element.

The BEM algorithm was implemented and validated by applying it to a cylindrical circular borehole filled with an inviscid fluid, for which the solution is known in closed form [48].

The results (displacements and pressures) in the time domain are obtained by simulating a Ricker wavelet pulse. This wavelet form is chosen because it decays rapidly, in both time and frequency, reducing the computational effort and allowing easier interpretation of the computed signatures.

As stated before, the computations are performed using complex frequencies of the form $\omega_{\mathrm{c}}=\omega-i \eta$ (with $\eta=0.7 \Delta \omega)$. In the time domain, the effect of the complex part of the frequencies is taken into account by applying an exponential window $\mathrm{e}^{\eta t}$ to re-scale the response [49].

\section{Dispersion of waves in a fluid-filled circular borehole}

Non-dispersive body waves, the dilatational (P) and shear (S) waves, as well as various types of guided waves, propagate along the interface between the fluid and solid. When the source is positioned on the axis of the cylinder, only the axisymmetric modes are excited. Additional modes with azimuthal variation are excited when the source is placed away from the axis. However, these later modes do not contribute to the pressure on the axis. Certain modes reveal the existence of cut-off frequencies, that is, they only exist if the source excitation frequency exceeds these frequencies.

The ratio between the shear wave velocity in the solid medium and the dilatational wave velocity in the fluid define two distinct modes of wave propagation. If the formation is slow (i.e. $\beta / \alpha_{f}<1.0$ ), proper normal modes do not exist, because any waves propagating in the fluid will radiate and lose their energy as shear waves in the solid. Leaky modes still exist, however, but they attenuate rapidly as they propagate. If the formation is fast (i.e. $\beta / \alpha_{f}>1.0$ ), the energy remains trapped in the fluid and normal modes exist in addition to the leaky modes. The amplitude of the normal modes in the elastic formation decays exponentially with the distance to the cylinder.

The work presented in this article simulates the cross-hole technique, for which two boreholes, with radii of $0.60 \mathrm{~m}$, are used and the effect of non-circular boreholes is addressed. However, the results obtained for a single circular borehole, with source and receivers placed within the fluid, are used as a reference. Two different formations, similar to the ones studied by Ellefsen [50], are used with the mechanical properties listed in the Table 1.

Figs. 2-3 display the phase and group velocities for the first normal modes in the fast and slow formation. The various modes are labeled by a pair of numbers. The first number reflects the azimuth order, while the second indicates the radial order that supplies the variation of the mode with radial distance.

The first axisymmetric mode is the tube wave or Stoneley wave $[(0,0)]$. It exists for all frequencies and its phase and group velocity values are below the dilatational velocity of the fluid. It exhibits slight dispersion, which is greater in the slow formation than in the fast formation.

The first mode with azimuth variation is the flexural wave $[(1,0)]$, which exists at all frequencies and is highly dispersive. However, the flexural wave is more dispersive in the fast formation than in the slow formation.

The second mode with azimuthal variation of $\cos 2 \theta$ or $\sin 2 \theta$, is the screw wave [(2,0)]. This screw wave has a cutoff frequency of approximately $1.0 \mathrm{kHz}$ in the fast formation and $0.7 \mathrm{kHz}$ in the slow formation. Higher radial modes exist in a fast formation.

The second axisymmetric mode is the pseudo-Rayleigh
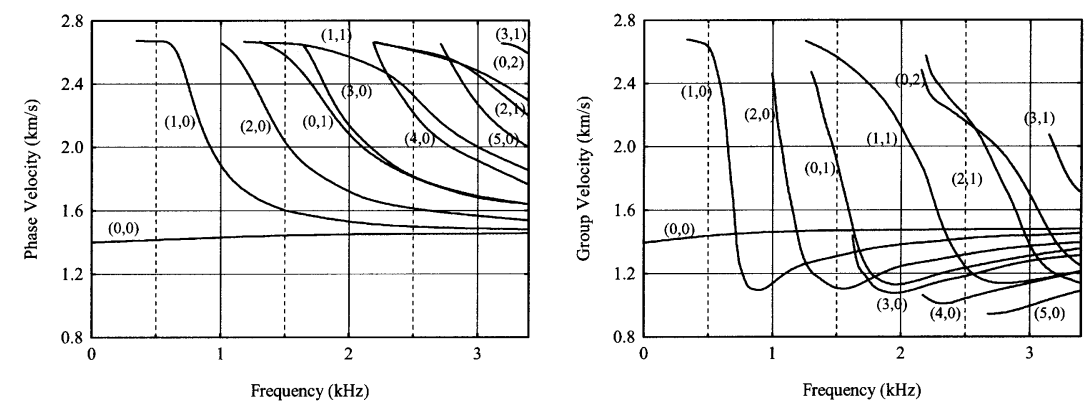

Fig. 2. Fast formation: phase and group velocities of the lowest normal modes. 

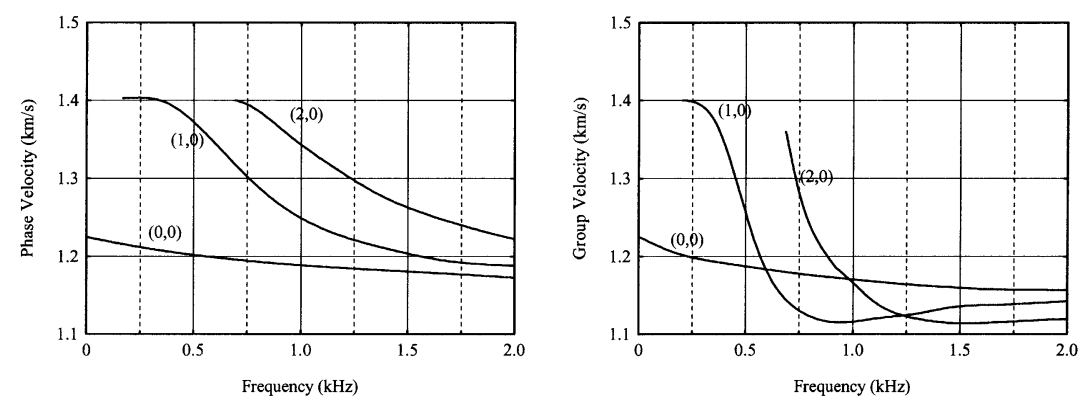

Fig. 3. Slow formation: phase and group velocities of the lowest normal modes.

wave $[(0,1)]$, which has a cut-off frequency of $1.35 \mathrm{kHz}$ in the present case. The mode reaches its highest phase velocity at this frequency, the shear wave velocity of the solid, and approaches the dilatational fluid velocity asymptotically as the frequency of the wave increases. On the other hand, the group velocity value is less than the shear wave velocity of the solid at a cut-off frequency. As the frequency increases, its velocity decreases rapidly to reach the minimum associated with Airy waves, and thereafter approaches the fluid velocity from below.

Higher flexural modes also exist, such as the $[(1,1)]$ and $[(2,1)]$ modes shown in Fig. 2. Higher order normal modes $[(n, m)]$ for $m, n>2$, which vary as $\cos n \theta$ or $\sin n x$, also exist, but they do not have a specific name.

\section{Synthetic Waveforms}

Synthetic waveforms have been computed to simulate the wave propagation between two fluid-filled cylindrical boreholes, driven parallel to the $z$ axis. The source is placed in the first borehole and the receivers are placed in the second borehole, on five planes, equally spaced $(6 \mathrm{~m})$ along the $z$ direction. The source is placed either on the axis of the borehole (source 1) or close to the wall of the borehole (source 2).

The BEM computations are performed using a number of boundary elements to discretize each borehole, which changes with the excitation frequency of the harmonic load. The ratio of the wavelength of the incident waves to the length of the boundary elements is kept at a minimum of 8.0. However, a minimum of 30 boundary elements is used to model each inclusion.

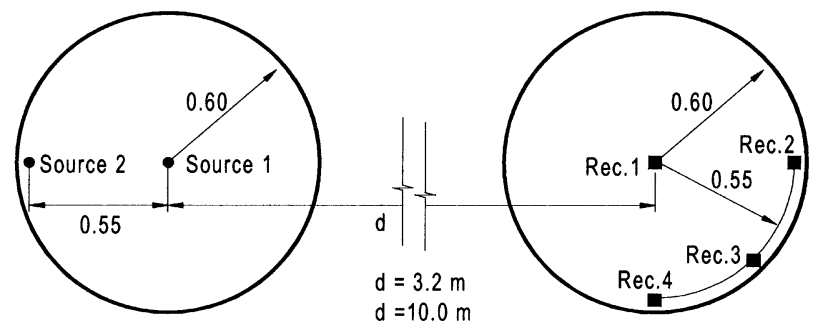

Fig. 4. Geometry of two cylindrical circular fluid-filled boreholes.
When the formation is fast, the computations are performed in the frequency range from 12.5 to $1600 \mathrm{~Hz}$, with a frequency increment of $12.5 \mathrm{~Hz} . T=1 / 12.5=$ $80 \mathrm{~ms}$ is the time thus allowed for the analysis, with the spatial distance between virtual sources set to $L=2 T \alpha_{s}=673 \mathrm{~m}$. The pressure time responses are computed modeling a spherical dilatational Ricker pulse source with a characteristic frequency $500 \mathrm{~Hz}$. Meanwhile, when the elastic formation is slow, the responses are calculated in the frequency range from 9.5 to $1216 \mathrm{~Hz}$, with a frequency increment of $9.5 \mathrm{~Hz} \quad(T=105.2 \mathrm{~ms}$ and $L=2 T \alpha_{s}=554 \mathrm{~m}$ ). The source time dependence is again a Ricker wavelet, but it now has a characteristic frequency of $450 \mathrm{~Hz}$.

In the first set of examples computed, the two boreholes are assumed to have circular cross-sections. Two distances between the two boreholes are selected, and these are 3.2 and $10.0 \mathrm{~m}$ (see Fig. 4). The results obtained are compared with those found when only one borehole is used for both the receivers and the source. The second set of examples simulates the presence of a non-circular cross-section borehole, an oval with an ovality ratio of $\epsilon=1.44 / 0.92=1.56$, which has the same perimeter as the neighboring circular borehole $(1.2 \pi \mathrm{m})$, as in Fig. 5 .

\subsection{Circular boreholes in a fast formation}

Figs. 6-9 show the response obtained when circular cross-section boreholes are driven in a fast formation, as in Fig. 4. Both the time and their Fourier Spectra responses are displayed, to give a better visual separation of the different wave types.

Fig. 6 displays the signal produced by the source 1, placed on the axis, acquired at receiver 2 , located close to the borehole wall. In a fast formation, the $\mathrm{P}$ wave begins as a dilatational wave in the borehole fluid; it is critically refracted into the formation as a $\mathrm{P}$ wave and is then refracted back into the fluid as a dilatational wave. The so-called $\mathrm{S}$ wave begins as a dilatational wave in the borehole fluid; it is critically refracted into the formation as an S wave, and is refracted into the fluid as a dilatational wave. After the $\mathrm{P}$ wave arrival, the response is marked by a dense ring called the leaky or PL mode. The scaling of the plots shown in 

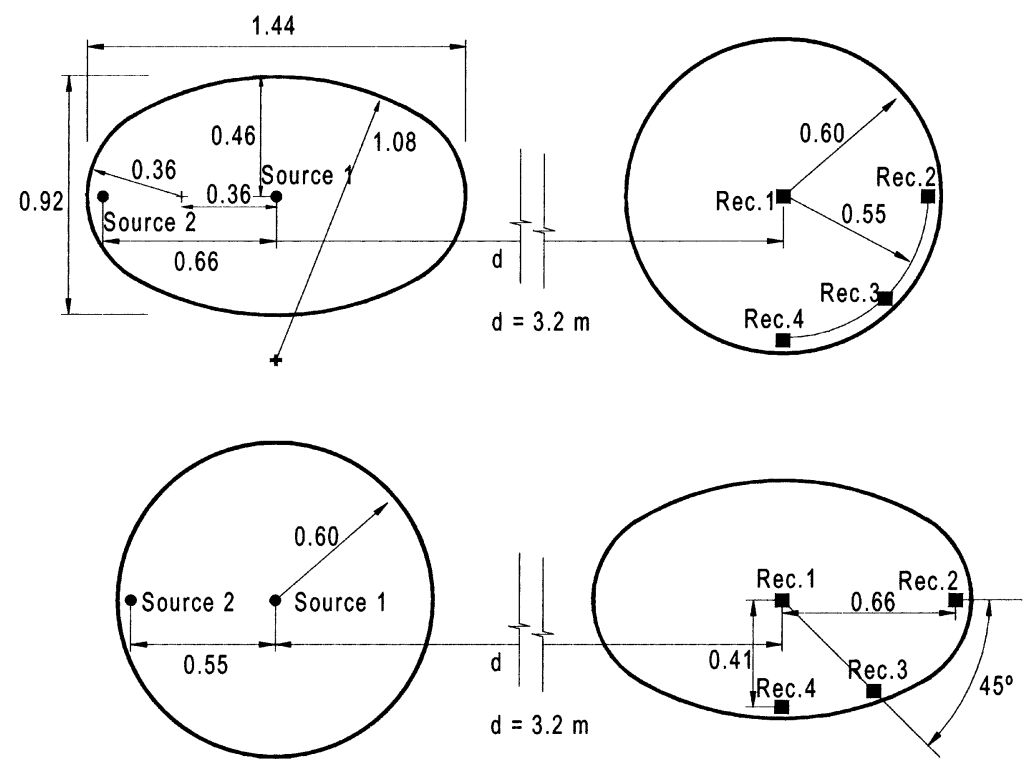

Fig. 5. Geometry of two cylindrical fluid-filled boreholes. (a) The source placed inside an oval while the receivers are placed in a circular borehole. (b) The source placed inside a circle while the receivers are placed in an oval borehole.

Fig. 6 does not allow easy observation of the P and S body waves.

When only one borehole, hosts both the source and the receiver, and the source is placed on its axis, axisymmetric normal modes alone are excited. The resulting guided waves, pseudo-Rayleigh and Stoneley waves, arrive after the $\mathrm{S}$ wave. The first pseudo-Rayleigh mode makes a very small contribution, given the frequency of the pulse excited $(500 \mathrm{~Hz})$, which is much lower than the cut-off frequency of these waves $(1.35 \mathrm{kHz})$. The result is a domination of the time responses by the Stoneley waves, which are only slightly dispersive, as the time plots in Fig. 6a illustrate. The features of the responses at the other receivers are similar (not shown).

When the source and receivers are placed in separate boreholes (cross-hole model), the behavior of the response changes markedly. Non-axisymmetric modes are excited, since the axis of the borehole, where the load is placed, is no longer axisymmetric. When the distance between boreholes is relatively small, $d=3.2 \mathrm{~m}$, the Stoneley waves still dominate the response, but now both the Flexural and the Screw waves are excited. Given the cut-off frequency of the Screw waves $(1.0 \mathrm{kHz})$, their contribution to the time response is small. However, the guided waves are less important, compared with the previous example (see Fig. $6 \mathrm{a}$ and $\mathrm{b}$ ), and the $\mathrm{S}$ wave starts to be clearly visible. Other body waves exist, caused by the multiple reverberations between the two boreholes. As we move the boreholes further apart, the guided waves continue to lose importance, as the spectra responses in Fig. 6c show. However, the Stoneley waves are still clearly visible at the low excitation frequencies. The time responses agree with this behavior by eliminating the late arrivals and by enhancing the presence of the $\mathrm{P}$ and $\mathrm{S}$ waves, which are now seen easily. The $\mathrm{P}$ mode converted waves, resulting from the incidence of $\mathrm{S}$ waves (originated on refraction into the formation of the dilatational waves within the fluid filled borehole) on the boreholes, are particularly clear. These pulses are identified in the plots with the labels SP. The Stoneley waves are not clearly observable given the frequency of the pulse excited $(500 \mathrm{~Hz})$. At low frequencies a Stoneley pulse should appear. To illustrate this behavior Fig. $6 \mathrm{c}$ also includes the responses obtained when the source excites a pulse with a frequency of $200 \mathrm{~Hz}$. As expected, a Stoneley pulse is now well visible.

Figs. 7-9 show the response generated by source 2, located close to the borehole wall. Fig. 7a displays the response at receiver 1, placed on the axis of one circular borehole. A source placed away from the axis excites both axisymmetric and non-axisymmetric modes, but the latter make a null contribution to the pressure on the axis of the borehole. Thus, only the body waves, the Stoneley waves $[(0,0)]$, and the pseudo-Rayleigh waves $[(0,1)]$ are given in Fig. 7a. The response there is similar to what would be created by a first source on the axis, except that the importance of the Stoneley wave is greater than for centered sources. The arrival times of the different pulses agree with the velocities of the $\mathrm{P}$ and $\mathrm{S}$ body waves, as well as with the phase defined above and the group velocity curves of the Stoneley and the pseudo-Rayleigh waves. The scaling of the plot shown in Fig. 7a does not allow easy observation of the $\mathrm{P}$ and $\mathrm{S}$ body pulses. In addition, the time plots do not reveal the existence of the pseudo-Rayleigh pulses, given the low characteristic frequency of the excited Ricker pulse $(500 \mathrm{~Hz})$, which is much lower than the cut-off frequency for these waves $(1.35 \mathrm{kHz})$.

If we move from the one circular borehole situation to the model with two boreholes, the spectra plots reveal the 


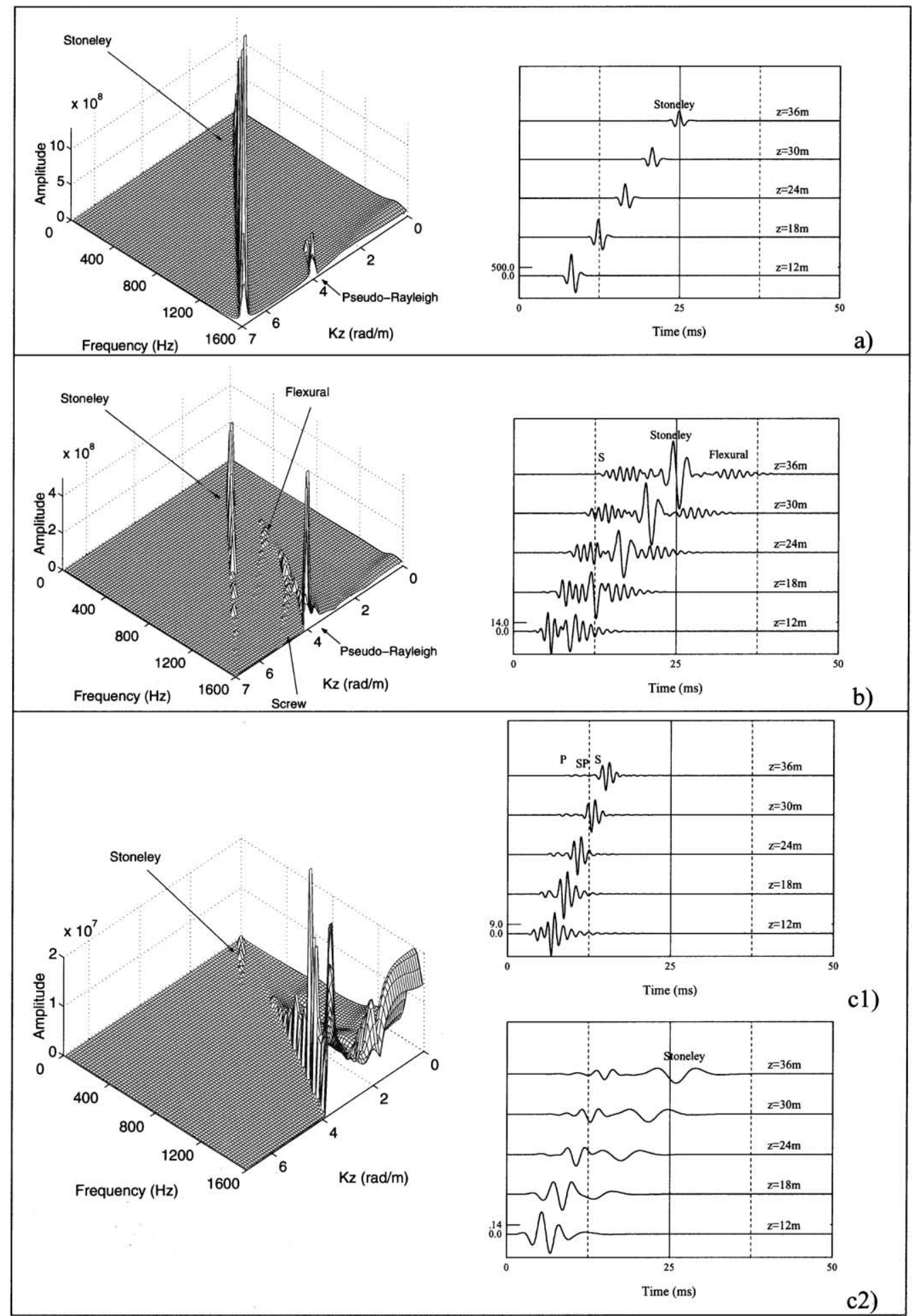

Fig. 6. Responses at receiver 2 when source 1 is excited (fast formation). (a) Circular borehole. (b) Two circular boreholes $(d=3.2 \mathrm{~m})$. (c) Two circular boreholes $(d=10.0 \mathrm{~m})$ : (1) Characteristic frequency of source $500 \mathrm{~Hz}$; (2) Characteristic frequency of source $200 \mathrm{~Hz}$.

existence of more modes (see Figs. $7 \mathrm{~b}$ and c). Two additional modes are clearly visible when the distance between boreholes is relatively small, namely the first order Flexural $[(1,0)]$ and the first order Screw modes $[(2,0)]$ (see Fig. $7 b)$. This behavior was expected since the axis of the borehole hosting the source is not an axisymmetric axis, and receiver 1 is no longer on the neutral axis of this dynamic system, which would inhibit the presence of the Flexural wave. The amplitudes of these modes, however, are not pronounced in comparison with the earlier modes: the Stoneley and the 


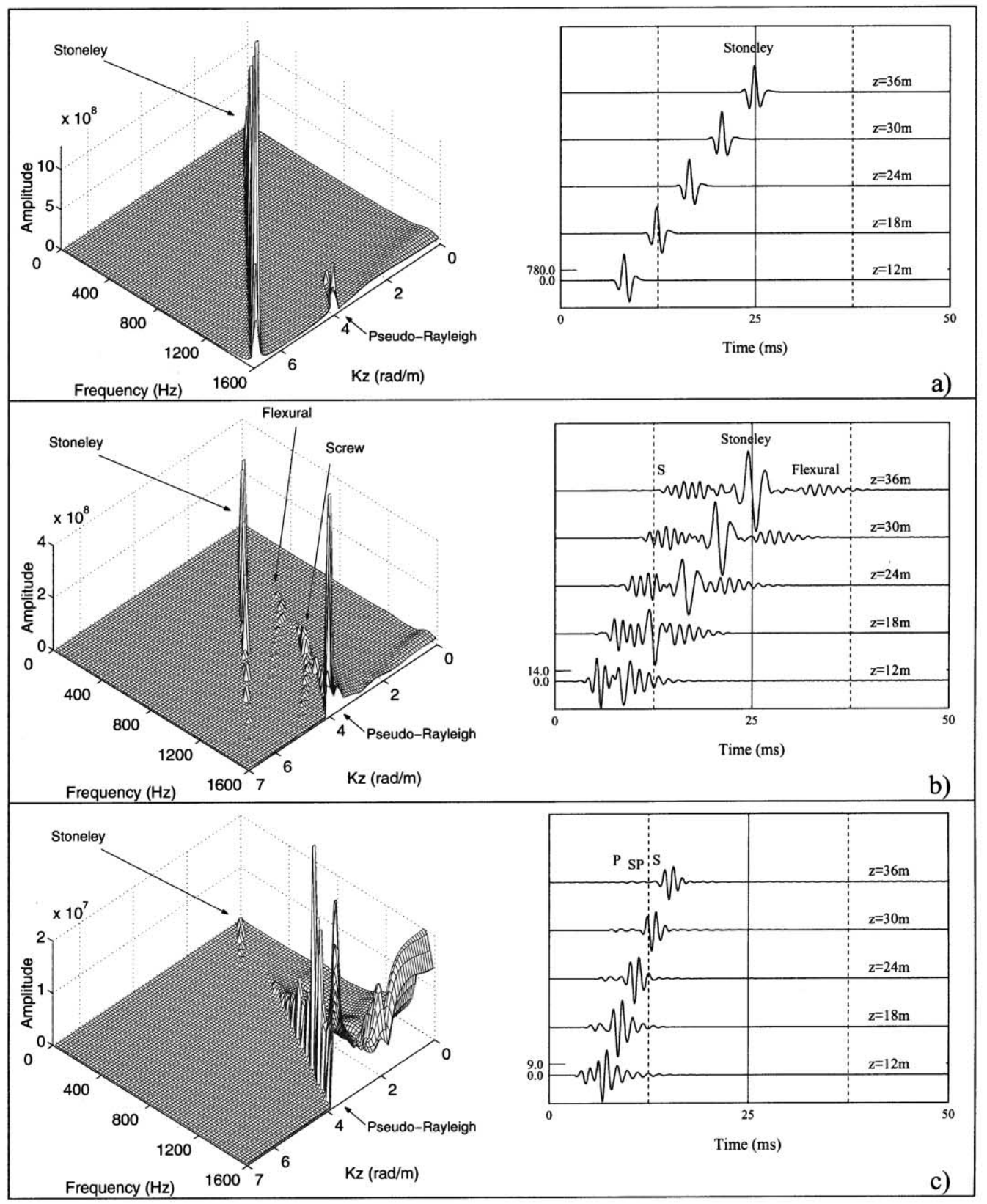

Fig. 7. Responses at receiver 1 when source 2 is excited (fast formation). (a) Circular borehole. (b) Two circular boreholes $(d=3.2 \mathrm{~m})$. (c) Two circular boreholes $(d=10.0 \mathrm{~m})$.

pseudo-Rayleigh modes. The time plots confirm this behavior by placing additional pulse trains after the arrival of the Stoneley wave. Notice that the Screw waves make a weak contribution to the response, because of its cut-off frequency $(1.0 \mathrm{kHz})$. The $\mathrm{S}$ body waves are now clearly visible, owing to the decrease in amplitude of the guided waves. As the second borehole is placed further away from the first, the guided modes decrease in amplitude and their contribution to the response is almost reduced to that of the Stoneley waves (see the spectra plot in Fig. 7c). The time responses agree with this behavior by enhancing the presence of the $\mathrm{P}$, $\mathrm{SP}$ and $\mathrm{S}$ body pulses.
Fig. 8 illustrates the response close to the wall of the borehole, at receiver 2 , which is placed in the same azimuth direction as the source. The responses at this receiver include contributions from the Flexural and Screw waves, as well as from the body, Stoneley and pseudo-Rayleigh waves.

As we have mentioned, the non-axisymmetric guided waves for one circular borehole are mainly caused by the first order Flexural waves $[(1,0)]$ since the Screw waves $[(2,0)]$ have a cut-off frequency of approximately $1 \mathrm{kHz}$. If we take the spectral representation of the response (see Fig. 8a), we can separate the different wave types visually. 


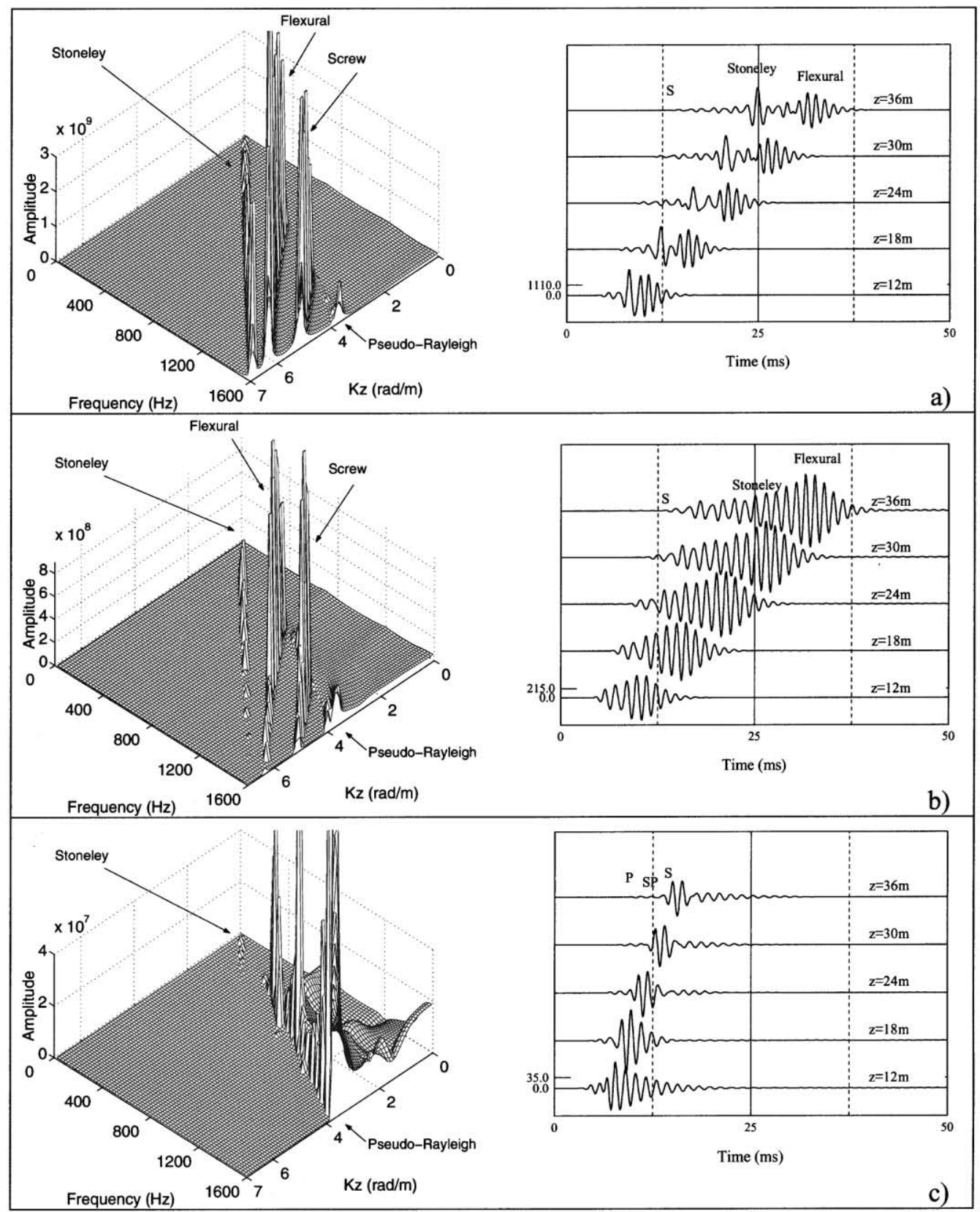

Fig. 8. Responses at receiver 2 when source 2 is excited (fast formation). (a) Circular boreholes. (b) Two circular boreholes $(d=3.2 \mathrm{~m})$. (c) Two circular boreholes $(d=10.0 \mathrm{~m})$.

This indicates that the group velocity of bending waves is higher than that of Screw waves, which in turn is higher than that for pseudo-Rayleigh waves. The amplitude of the Stoneley pulse in the time domain response is slightly bigger than its amplitude on the axis (receiver 1), as would be expected.

As we move from one borehole to two boreholes, we notice that the guided waves lose their importance (see Figs. 8a-c). Furthermore, it can be observed that the Stoneley waves become less important in relation to the other guided modes, when the distance between boreholes is set to $d=3.20 \mathrm{~m}$. When the distance between boreholes further increases $(d=10.0 \mathrm{~m})$, the responses show the same behavior as that found at receiver 1 , that is, the response is mainly produced by the body waves. In the time plots, this is shown by the progressive disappearance of late arrivals, after the $\mathrm{S}$ arrival.

The signatures at receiver 4 are not affected by Flexural waves when there is only one borehole. This is because the bending mode has zero amplitude at the neutral axis, which is the vertical plane through the axis that is perpendicular to the line connecting the center and the source. Hence, after the passage of the Stoneley wave, the signatures on the neutral axis experience a substantial drop in amplitude 


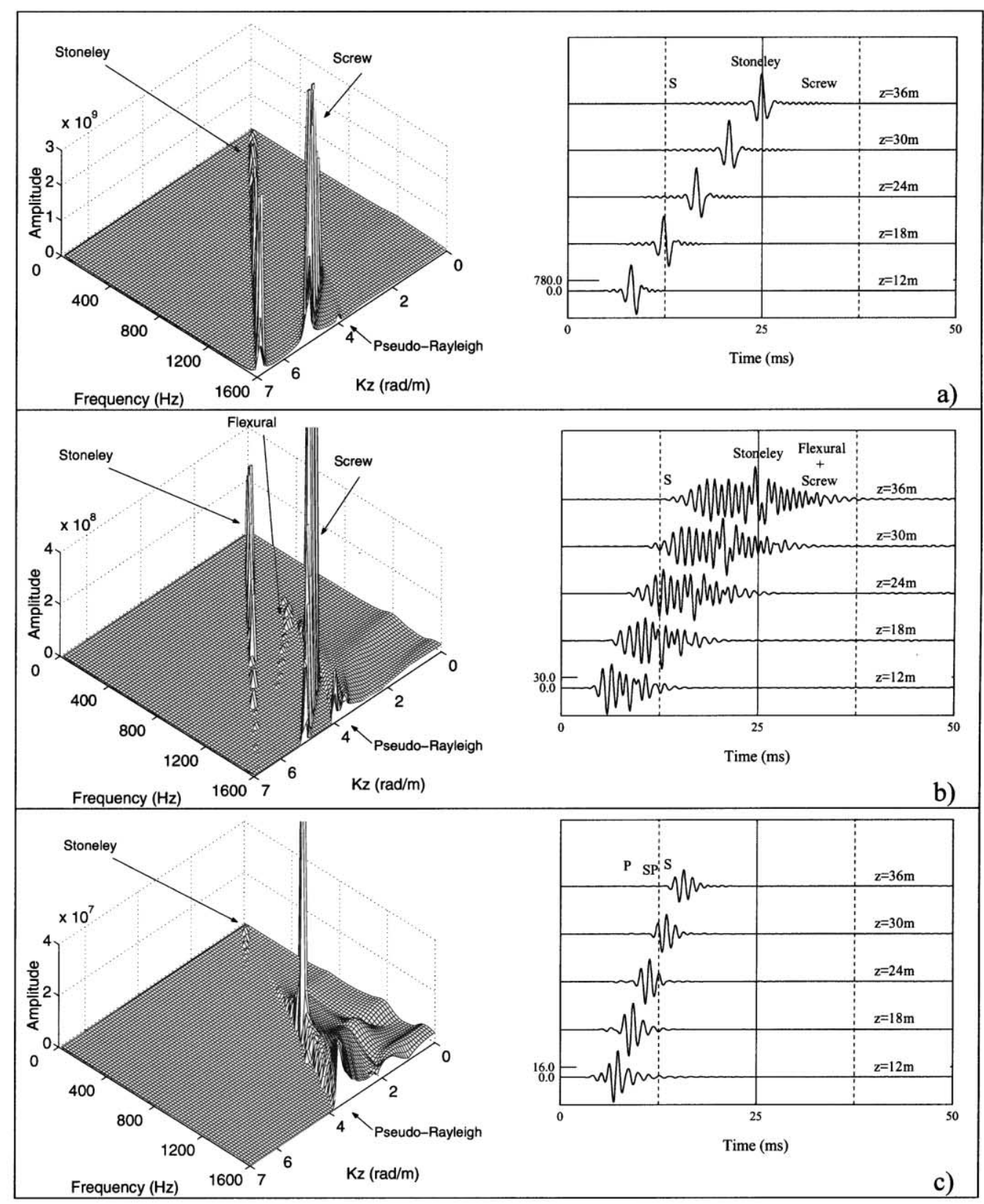

Fig. 9. Responses at receiver 4 when source 2 is excited (fast formation). (a) Circular borehole. (b) Two circular boreholes $(d=3.2 \mathrm{~m})$. (c) Two circular boreholes $(d=10.0 \mathrm{~m})$.

(Fig. 9a). This drop is not, however, observed for receivers 2 (Fig. 8a), and 3 (not shown) located away from the neutral axis. Indeed, receiver 2 , which is farthest from the neutral axis, exhibit the largest increases in pressure, which are clearly due to the flexural mode. At receiver 4, when there is only one borehole, the only surviving guided waves are those coming from the Screw mode (see the frequency spectra plot in Fig. 9a). As for receiver 2, the guided waves lose importance when the two-borehole model is used. When the distance between boreholes is $d=3.20 \mathrm{~m}$, the Stoneley wave is still visible. When this distance is increased to $d=10.0 \mathrm{~m}$, the response is almost solely due to the contribution of the body waves.

\subsection{Circular boreholes in a slow formation}

Fig. 10 shows the responses generated by source 2 at receiver 2, when the boreholes are inside a slow formation. As expected, the responses obtained at the receivers away from the axis include contributions from the non-axisymmetric modes. Given the nature of a slow formation, only the first order normal modes exist, as pointed out before. The weak contrast among the group velocities of the different modes and the central frequency used to generate the time series, does not allow the contributions of the various wave types to be isolated. It is nevertheless possible to 


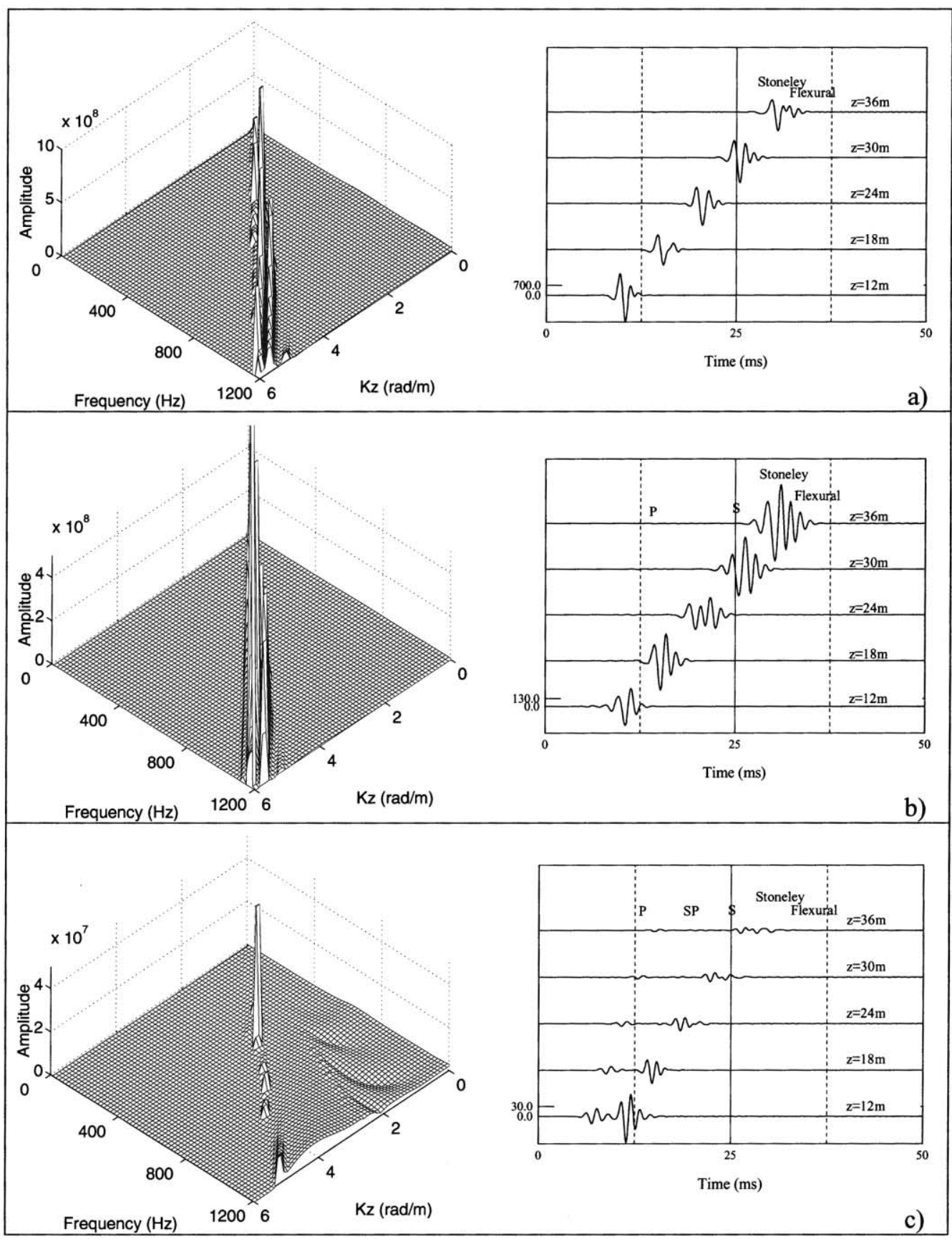

Fig. 10. Responses at receiver 2 when source 2 is excited (slow formation). (a) Circular borehole. (b) Two circular boreholes $(d=3.2 \mathrm{~m})$. (c) Two circular boreholes $(d=10.0 \mathrm{~m})$.

detect the presence of the Stoneley wave when only one borehole is used to allocate both the source and receivers (see Fig. 10a). Once more, as we move from one circular borehole to two circular boreholes, the guided waves decrease in importance. When the distance between boreholes reaches $d=10.0 \mathrm{~m}$, the body waves begin to dominate the response. Again, the P mode converted waves resulting from the incidence of $\mathrm{S}$ waves (produced by the refraction of the dilatational waves within the fluid filled borehole into the formation) on the boreholes are well visible and labeled SP.

\subsection{Non-circular boreholes in a fast formation}

The next application illustrates how the solution changes when one of the boreholes suffers a wall deformation and becomes non-circular. Two different models are used to define the main wave propagation differences. In the first case, the source is placed inside a fluid-filled borehole with an oval cross-section, and the receivers are located in a second borehole with a circular cross-section. In the second case, the circular borehole is used to host the source while 


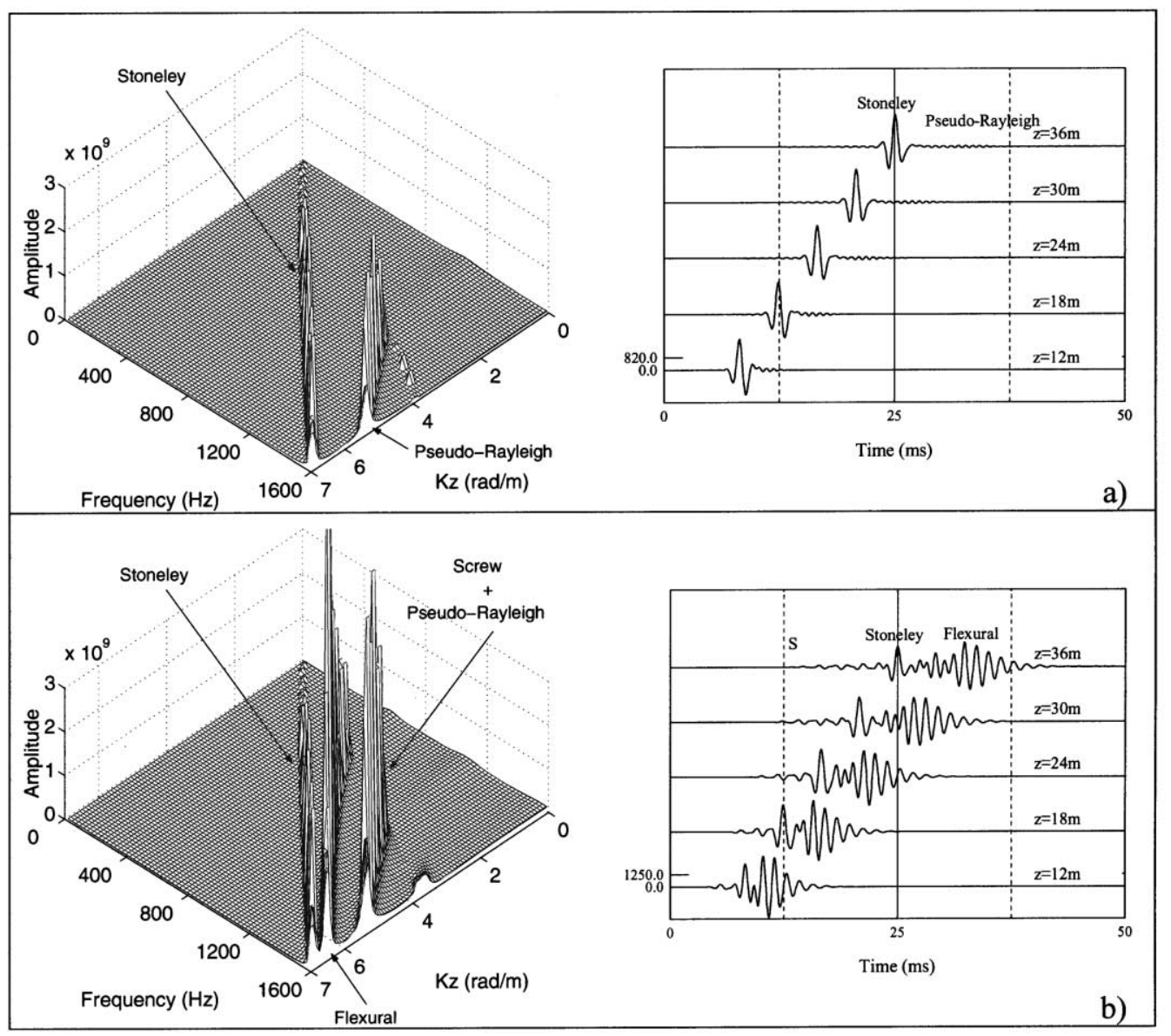

Fig. 11. Responses when source 2 is excited (fast formation) inside an oval fluid-filled borehole. (a) Receiver 1. (b) Receiver 2.

the oval borehole contains the receivers (see Fig. 5). The distance between borehole axes is constant $(d=3.20 \mathrm{~m})$.

The responses obtained for one fluid-filled borehole with an oval cross-section, hosting both the source and receivers, are used as a reference. Fig. 11 displays the responses obtained when the source 2, placed close to the borehole wall, is excited. The spectra response obtained at receiver 1 , placed on the axis, exhibits a slower Stoneley wave at lower frequencies than was found for the single circular borehole (see Figs. 11a and 7a). The time plots confirm this phenomenon by attributing a slightly delayed arrival to the Stoneley pulses. This effect agrees with the tendency found by Randall [19] when calculating the dispersion curves for modes of noncircular fluid-filled boreholes for low frequencies, and later confirmed by the authors Tadeu et al. [39] in the calculation of time series for oval fluid-filled boreholes. In addition, as we move from the circular to the oval, our results indicate that the amplitude of the Stoneley pulses increases. Furthermore, it is observed that the pseudo-Rayleigh cut-off frequency decreases as the ovality ratio increases. A second order pseudo-Rayleigh $[(0,2)]$ can even be observed. The time responses reflect the presence of these waves, exhibiting a ring of pulses after the $S$ wave arrives, which decreases rapidly, given the low frequency of the pulse excited.
Fig. 11b illustrates the response at receiver 2, close to the wall of the borehole, placed in the same azimuth direction as the source. Flexural and Screw waves are now present, in addition to the body, Stoneley and pseudo-Rayleigh waves. As we shift our attention from the circular inclusion to the oval, we notice that the flexural waves reach lower velocities at lower frequencies, approaching the velocity (found previously, for the circular inclusion) for later frequencies, as the ovality ratio increases. In the time plots, this can be seen in the progressive appearance of late arrivals, after the Stoneley wave. The Screw wave behaves in a similar way, but, in addition, it rapidly loses importance.

When the simulation uses two boreholes, non-axisymmetric normal modes are excited as expected, and they follow the behavior described above. However, the responses become more complex since there are several normal modes excited by both the circular and the oval boreholes, with distinct phase and group velocities. This leads to spectral responses that are more difficult to analyze, given the increased number of mode behaviors. To allow easier interpretation, the spectra plots are now presented as gray scale plots, which consequently lose the third dimension aspect, but which permit the different mode patterns to be distinguished visually. 

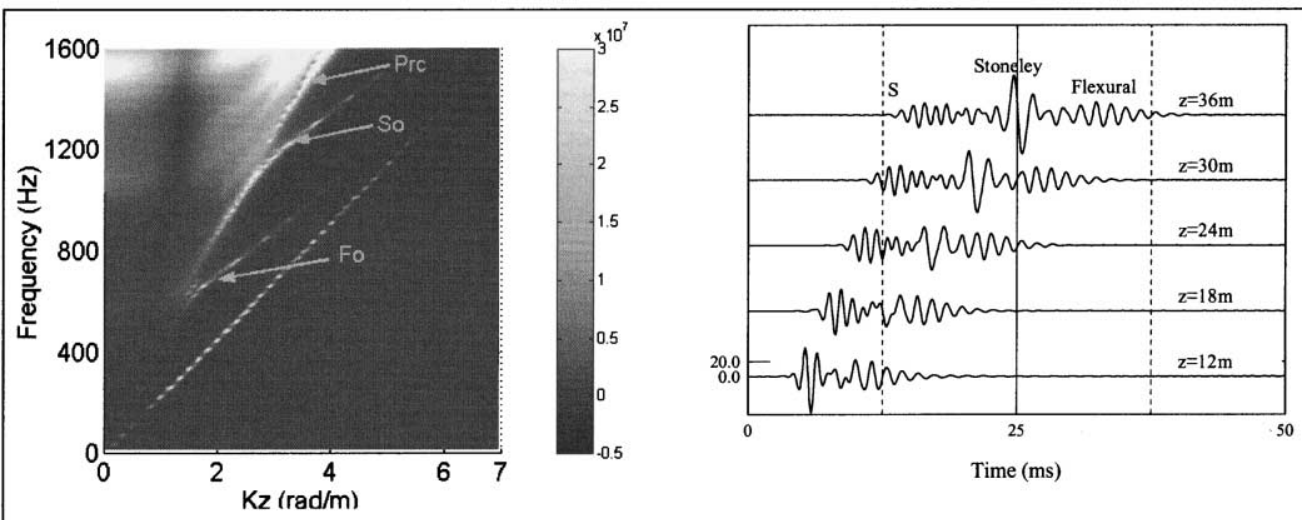

Prc - Pseudo-Rayleigh excited by the circular borehole

So - Screw mode excited by the oval borehole

Fo - Flexural mode excited by the oval borehole

a)

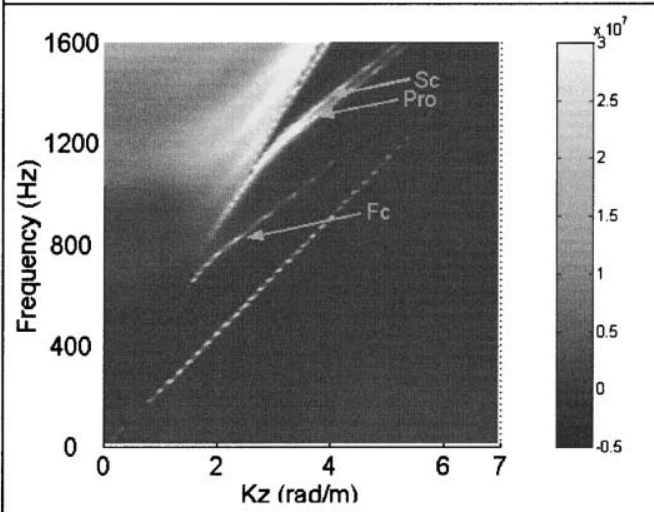

Pro - Pseudo-Rayleigh excited by the oval borehole

Sc - Screw mode excited by the circular borehole

Fc - Flexural mode excited by the circular borehole

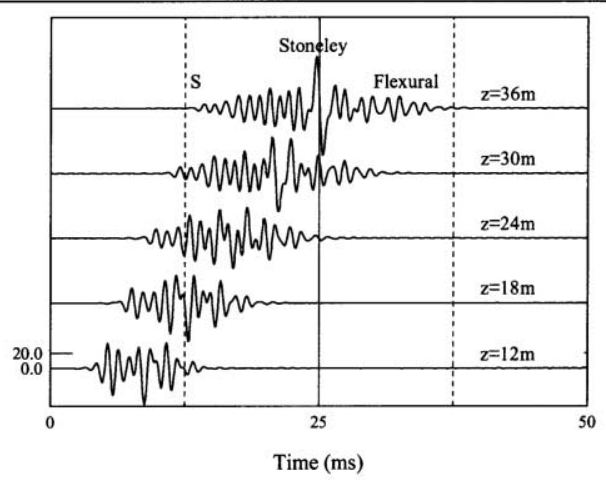

b)

Fig. 12. Responses at receiver 1 when source 2 is excited (fast formation). (a) The borehole hosting the source is oval while the borehole allocating the receiver is circular. (b) The borehole hosting the source is circular while the borehole allocating the receiver is oval.

Fig. 12 displays the computed results when source 2 is allocated to one borehole and a second borehole hosts the receiver 1 . As before, the guided waves amplitude suffers a pronounced decay when compared with the response for one borehole, hosting both the source and receiver. Consequently, the $S$ wave arrivals are observable in the time plots. The spectral response, calculated when the oval borehole hosts the source, registers the presence of both the Flexural and the Screw waves excited by the oval borehole, in addition to the Stoneley and pseudo-Rayleigh waves excited by the circular borehole (Fig. 12a). When the source is placed in the circular borehole, the results reveal similar behavior. However, the Flexural and the Screw waves originate within the circular inclusion, while the Stoneley and pseudo-Rayleigh waves are the ones excited by the oval borehole (Fig. 12b). The time results reflect this behavior by enhancing the amplitude of the Stoneley wave when the receiver is inside the oval inclusion (Fig. 12b), and by placing pulses later in time when the source is in the oval inclusion (Fig. 12a), which was expected, given the behavior described for an oval borehole.

Fig. 13 shows the computed responses when one borehole hosts source 2, with the second borehole hosting receiver 2, placed close to the borehole wall. The results reveal the presence of non-axysimmetric normal modes, excited by both the oval and the circular boreholes, because receiver 2 is placed away from the axes of the two boreholes. Thus, two sets of Screw and Flexural wave modes are given in the spectral plots when the source is placed either within the circular or within the oval borehole. The time plots display similar behavior as the time progresses. Both time results register a weaker Stoneley wave.

When these results are compared with those generated by the two circular boreholes, our results reveal that the amplitude of the guided waves has now decreased. This phenomenon can be explained by the fact that in the case of the circular boreholes, both the source and receiver hosting boreholes produce non-axisymmetric waves that have the 

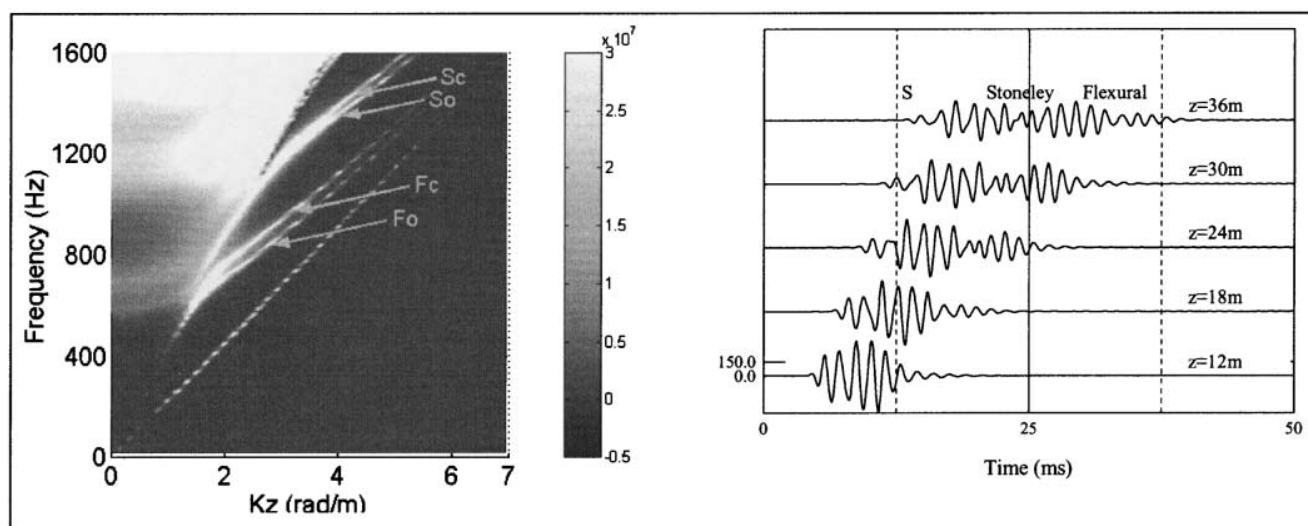

Sc - Screw mode excited by the circular borehole So - Screw mode excited by the oval borehole Fc - Flexural mode excited by the circular borehole

a)

Fo - Flexural mode excited by the oval borehole
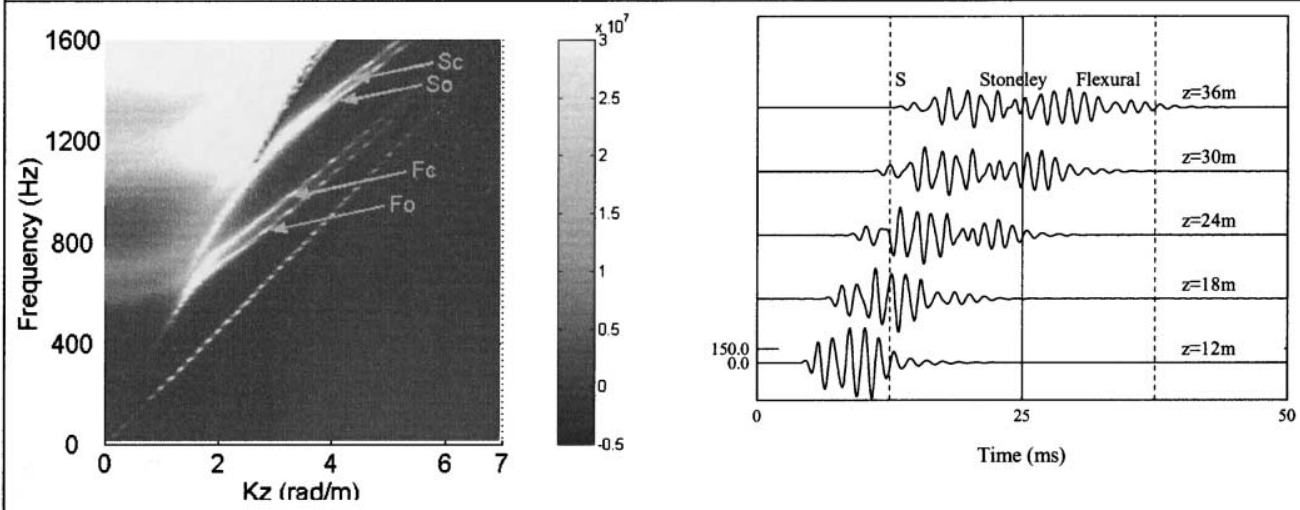

Sc - Screw mode excited by the circular borehole So - Screw mode excited by the oval borehole Fc - Flexural mode excited by the circular borehole Fo - Flexural mode excited by the oval borehole

Fig. 13. Responses at receiver 2 when source 2 is excited (fast formation). (a) The borehole hosting the source is oval while the borehole allocating the receiver is circular. (b) The borehole hosting the source is circular while the borehole allocating the receiver is oval.

same group and phase velocities, which enhances the amplitude of the guided modes (see Figs. 8 and 13). This superposition of waves, originating in normal modes with the same azimuth and radial order, does not occur for the same group and phase velocities when the boreholes have different geometries.

\section{Conclusions}

The BEM was developed and implemented to evaluate the 3D wave field generated by a dilatational point load illuminating a physical system constituted by either one or two fluid-filled boreholes placed inside an elastic formation. This model was built so that it simulated the cross-hole seismic testing technique where the source is placed within one borehole and the receivers are placed in a neighboring borehole. Different models were analyzed, assuming both circular and non-circular (oval) cross-sections for the boreholes. These analyses were used to assess the influence of the source and receiver positions and the distance between boreholes on the propagation of body waves, and on both axisymmetric and non-axisymmetric wave modes, in the presence of different borehole cross-sections. Both the time responses and their Fourier Spectral representations were included to give better identification of the various wave components.

Analyses reveal that, as we move from a single circular borehole to a system where the source and the receivers are located in different circular boreholes, marked changes occur in the responses, due mainly to a highly enhanced contribution by non-axisymmetric normal modes. This enhancement is particularly visible for receivers that were previously located on the axis of the one borehole system, which constitutes the axisymmetric axis of the dynamic system. For the case of two boreholes, the source is no 
longer on the axisymmetric axis of the system, and thus the nonaxisymmetric modes, such as the Flexural and the Screw modes excited in the first borehole, become increasingly important. Additionally, it may be observed that, as the two boreholes are separated by greater distances, both the frequency spectra and the time responses reveal an increased importance of the body waves ( $\mathrm{P}, \mathrm{SP}$ and $\mathrm{S}$ ), and a gradual loss of the contribution of the guided modes to the response. The Stoneley wave, however, is still clearly visible, as expected.

When the two boreholes have different cross-sections the responses become more complex, owing to the excitation of normal modes by different phase and group velocities, which nevertheless have the same azimuth and radial order, originating within each borehole. Additionally, our results indicate a decrease in the response amplitude when the previously circular cross-section changes its shape to oval.

\section{Appendix A. The Green's functions}

\section{A.1. Solid formation}

Definitions

$\lambda, \mu \quad$ Lamé constant

$\rho \quad$ mass density

$\alpha=\sqrt{(\lambda+2 \mu) / \rho} \quad \mathrm{P}$ wave velocity

$\beta=\sqrt{\mu / \rho} \quad \mathrm{S}$ wave velocity

$k_{p}=\omega / \alpha, \quad k_{s}=\omega / \beta$

$k_{\alpha}=\sqrt{k_{p}^{2}-k_{z}^{2}}, \quad k_{\beta}=\sqrt{k_{s}^{2}-k_{z}^{2}}$

$A=\frac{1}{4 i \rho \omega^{2}} \quad$ amplitude

$\gamma_{i}=\frac{\partial r}{\partial x_{i}}=\frac{x_{i}}{r} \quad i=1,2 \quad$ direction cosines

$H_{n \alpha}=H_{n}^{(2)}\left(k_{\alpha} r\right), H_{n \beta}=H_{n}^{(2)}\left(k_{\beta} r\right) \quad$ Hankel functions

$B_{n}=k_{\beta}^{n} H_{n \beta}-k_{\alpha}^{n} H_{n \alpha} \quad B_{n}$ functions

Green's functions for displacements

$$
\begin{aligned}
& G_{x x}=A\left[k_{s}^{2} H_{0 \beta}-\frac{1}{r} B_{1}+\gamma_{x}^{2} B_{2}\right] \\
& G_{y y}=A\left[k_{s}^{2} H_{0 \beta}-\frac{1}{r} B_{1}+\gamma_{y}^{2} B_{2}\right] \\
& G_{z z}=A\left[k_{s}^{2} H_{0 \beta}-k_{z}^{2} B_{0}\right] \quad G_{x y}=G_{y x}=\gamma_{x} \gamma_{y} A B_{2} \\
& G_{x z}=G_{z x}=i k_{z} \gamma_{x} A B_{1} \quad G_{y z}=G_{z y}=i k_{z} \gamma_{y} A B_{1}
\end{aligned}
$$

Volumetric strain (super-index $=$ direction of load)

$$
\begin{aligned}
\epsilon_{\mathrm{Vol}}^{l} & =G_{x l, x}+G_{y l, y}+G_{z l, z} \\
& =A\left[\frac{\partial}{\partial x_{l}}\left(k_{s}^{2} H_{0 \beta}\right)+B_{0, x l x}+B_{0, y l y}+B_{0, z l z}\right] \\
& =A \frac{\partial}{\partial x_{l}}\left[k_{s}^{2} H_{0 \beta}+B_{0, x x}+B_{0, y y}+B_{0, z z}\right] \\
& =A \frac{\partial}{\partial x_{l}}\left[k_{s}^{2} H_{0 \beta}+\hat{\nabla}^{2} B_{0}\right]
\end{aligned}
$$

Note: $H_{0 \beta, l}=-k_{\beta} \gamma_{l} H_{l \beta}, H_{0 \beta, z}=-i k_{z} H_{0 \beta}$

Strain components (tensor definition, not engineering)

$$
\begin{aligned}
\epsilon_{i j}^{l} & =(1 / 2)\left(G_{i l, j}+G_{j l, i}\right) \\
& =(1 / 2) A\left(\delta_{i l} k_{s}^{2} H_{0 \beta, j}+\delta_{j l} k_{s}^{2} H_{0 \beta, i}+B_{0, i l j}+B_{0, j l i}\right) \\
& =(1 / 2) k_{s}^{2} A\left(\delta_{i l} H_{0 \beta, j}+\delta_{j l} H_{0 \beta, i}\right)+A B_{0, i j l}
\end{aligned}
$$

(a) Strains for loads in the plane, $l=x, y$

$$
\begin{aligned}
& \epsilon_{\mathrm{Vol}}^{l}=\gamma_{l} A\left(-k_{s}^{2} k_{\beta} H_{1 \beta}+k_{z}^{2} B_{1}+\frac{4}{r} B_{2}-B_{3}\right) \\
& \epsilon_{x x}^{l}=\gamma_{l} A\left(\left(\frac{2}{r} B_{2}-k_{s}^{2} k_{\beta} H_{1 \beta}\right) \delta_{x l}+\frac{1}{r} B_{2}-\gamma_{x}^{2} B_{3}\right) \\
& \epsilon_{y y}^{l}=\gamma_{l} A\left(\left(\frac{2}{r} B_{2}-k_{s}^{2} k_{\beta} H_{1 \beta}\right) \delta_{y l}+\frac{1}{r} B_{2}-\gamma_{y}^{2} B_{3}\right) \\
& \epsilon_{z z}^{l}=\gamma_{l} k_{z}^{2} A B_{1} \\
& \epsilon_{x y}^{l}=A\left(\left(\frac{1}{r} B_{2}-\frac{1}{2} k_{s}^{2} k_{\beta} H_{1 \beta}\right)\left(\delta_{x l} \gamma_{y}+\delta_{y l} \gamma_{x}\right)-\gamma_{x} \gamma_{y} \gamma_{l} B_{3}\right) \\
& \epsilon_{x z}^{l}=i k_{z} A\left(\left(\frac{1}{r} B_{1}-\frac{1}{2} k_{s}^{2} H_{0 \beta}\right) \delta_{x l}-\gamma_{x} \gamma_{l} B_{2}\right) \\
& \epsilon_{y z}^{l}=i k_{z} A\left(\left(\frac{1}{r} B_{1}-\frac{1}{2} k_{s}^{2} H_{0 \beta}\right) \delta_{y l}-\gamma_{y} \gamma_{l} B_{2}\right)
\end{aligned}
$$

(b) Strain for axial loads, $l=z$

$$
\begin{aligned}
& \epsilon_{\mathrm{Vol}}^{z}=i k_{z} A\left(-k_{s}^{2} H_{0 \beta}+k_{z}^{2} B_{0}+\frac{2}{r} B_{1}-B_{2}\right) \\
& \epsilon_{x x}^{z}=i k_{z} A\left(\frac{1}{r} B_{1}-\gamma_{x}^{2} B_{2}\right) \quad \epsilon_{y y}^{z}=i k_{z} A\left(\frac{1}{r} B_{1}-\gamma_{y}^{2} B_{2}\right) \\
& \epsilon_{z z}^{z}=i k_{z} A\left(-k_{s}^{2} H_{0 \beta}+k_{z}^{2} B_{0}\right) \quad \epsilon_{x y}^{z}=-i k_{z} \gamma_{x} \gamma_{y} A B_{2} \\
& \epsilon_{x z}^{z}=\gamma_{x} A\left(-\frac{1}{2} k_{s}^{2} k_{\beta} H_{1 \beta}+k_{z}^{2} B_{1}\right) \\
& \epsilon_{y z}^{z}=\gamma_{y} A\left(-\frac{1}{2} k_{s}^{2} k_{\beta} H_{1 \beta}+k_{z}^{2} B_{1}\right)
\end{aligned}
$$

(c) Stresses

$\tau_{i j}^{l}=\lambda \epsilon_{\mathrm{Vol}}^{l} \delta_{i j}+2 \mu \epsilon_{i j}^{l}$ 


\section{A.2. Fluid formation}

\section{Definitions}

$\lambda_{f} \quad$ Lamé constant

$\rho_{f} \quad$ mass density

$\alpha_{f} \quad$ P wave velocity

$k_{p f}=\omega / \alpha_{f}$

$k_{\alpha f}=\sqrt{k_{p f}^{2}-k_{z}^{2}}$

$A_{f}=\frac{1}{4 i} \quad$ amplitude

$\gamma_{i}=\frac{\partial r}{\partial x_{i}}=\frac{x_{i}}{r}, i=1,2 \quad$ direction cosines

$H_{n \alpha f}=H_{n}^{(2)}\left(k_{\alpha f} r\right) \quad$ Hankel functions

Green's functions for displacements

$G_{f x}=-A_{f} k_{\alpha f} H_{1 \alpha f} \gamma_{x} G_{f y}=-A_{f} k_{\alpha f} H_{1 \alpha f} \gamma_{y}$

Stresses

$H_{f 1}=A_{f} \lambda_{f} H_{0 \alpha f}\left(-\omega^{2} / \alpha_{f}^{2}\right)$

\section{References}

[1] Marzetta TL, Orton M, Krampe A, Johnston LK, Wuenschel PC. A hydrophone vertical seismic profiling experiment. Geophysics 1988;53:1437-44.

[2] Lee JM. In situ seismic anisotropy and its relationship to crack and rock fabrics. PhD thesis, Pennsylvania State University, 1990.

[3] Albright JN, Johnson PA. Cross-borehole observation of mode conversion from borehole Stoneley waves to channel waves at a coal layer. Geophys Prosp 1990;38:607-20.

[4] Krohn CE. Crosswell continuity logging using guided seismic waves. The Leading Edge 1992;11(7):39-45.

[5] Toksöz MN, Cheng CH, Cicerone RD. Fracture detection and characterization from hydrophone vertical seismic profiling data. In: Evans B, Wong T, editors. Fault mechanics and transport properties of rocks, London: Academic Press, 1992. p. 389-414.

[6] Findlay MJ, Goulty NR, Kragh JE. The crosshole seismic reflection method in open cast coal exploration. First Break 1991;9:09-14.

[7] Goulty NR, Thatcher JS, Findlay MJ, Kragh JE, Jackson PD. Experimental investigation of crosshole seismic techniques for shallow coal exploration. Q J Engng Geol 1990;23:217-28.

[8] Becquey M, Bernet-Rollande JO, Laurent J, Noual G. Imaging reservoirs - A crosswell seismic experiment. First Break 1992; 10:337-44.

[9] Lazaratos SK, Rector JW, Harris JM, Van Schaack M. Highresolution crosswell imaging of a West Texas carbonate reservoir: Part 4. Reflection imaging. 62nd. Annual International Meeting, Society of Exploration Geophysicists expanded abstracts, 1992; p. 49-53.

[10] Khalil AA, Stewart RR, Henley DC. Traveltime inversion and reflection processing of crosswell seismic data. Geophysics 1993;58:1248-56.

[11] Hardage BA. Crosswell seismology and reverse VSP. Geophysical Press, 1992.

[12] Rowbotham PS, Goulty NR. Wavefield separation by 3-D filtering in crosshole seismic reflection processing. Geophysics 1994;59:106571.
[13] Lee MW, Balch AH. Theoretical seismic wave radiation from a fluidfilled borehole. Geophysics 1982;47:1308-14.

[14] Lee MW, Balch AH, Parrot KR. Radiation from a downhole airgun source. Geophysics 1984;49:27-36.

[15] Lee MW. Low-frequency radiation from point sources in a fluid-filled borehole. Geophysics 1986;51:1801-7.

[16] Winbow GA. Seismic sources in open and cased boreholes. Geophysics 1991;56:1040-50.

[17] Stephen RA, Cardo-Casas F, Cheng CH. Finite difference synthetic acoustic logs. Geophysics 1985;50:1588-609.

[18] Bouchon M, Schmitt DP. Full wave acoustic logging in an irregular borehole. Geophysics 1989;54:758-65.

[19] Randall CT. Multipole acoustic waveforms in nonaxisymmetric boreholes and formations. J Acoust Soc Am 1991;90:1620-31.

[20] Leslie HD, Randall CT. Multipole sources in boreholes penetrating anisotropic formations: Numerical and experimental results. J Acoust Soc Am 1992;91:12-27.

[21] Yoon KH, McMechan GA. 3-D finite difference modelling of elastic waves in borehole environments. Geophysics 1992;57:793-804.

[22] Cheng N, Cheng CH, Toksöz MN. Borehole wave propagation in three dimensions. J Acoust Soc Am 1995;97:3483-93.

[23] Track A, Daube F. Borehole coupling in cross-well wave propagation. EAEG expanded abstracts, paper B026, Paris, 1992.

[24] Cheng N, Zhu Z, Cheng CH, Toksöz MN. Experimental and finite difference modeling of borehole Mach waves. EAEG expanded abstracts, paper P070, Paris, 1992.

[25] White JE, Sengbush RL. Shear waves from explosive sources. Geophysics 1963;28:1001-19.

[26] Heelan PA. Radiation from a cylindrical source of finite length Geophysics 1953;18:685-96.

[27] Ben-Menahem A, Kostek S. The equivalent force system of a monopole source in a fluid-filled open borehole. Geophysics 1990;56: $1477-81$.

[28] Kurkjian AL, Hon BP, White JE, De Hoop AT, Marzetta TL. A moving point mechanism representation for low frequency monopole borehole sensors. EAEG expanded abstracts, paper P074, Paris, 1992.

[29] Kurkjian AL, Schmidt H, White JE, Marzetta TL, Chouzenoux C. Numerical modeling of cross-well seismic monopole sensor data. 62nd Annual International Meeting, Society of Exploration Geophysicists expanded abstracts, 1992; p. 141-4.

[30] Gibson RL. Modeling mach wave propagation between boreholes in layered media. EAEG expanded abstracts, paper B027, Paris, 1992.

[31] De Hoop AT, de Hon BP, Kurkjian AL. Calculation of transient tube wave signals in cross-borehole acoustics. J Acoust Soc Am 1994; 95:1773-89.

[32] Kurkjian AL, Coates RT, White JE, Schmidt H. Finite-difference and frequency-wavenumber modeling of seismic monopole sources and receivers in fluid-filled boreholes. Geophysics 1994;59:1053-64.

[33] Peng C, Lee JM, Toksöz NM. Pressure in a fluid-filled borehole caused by a seismic source in stratified media. Geophysics 1996; 61:43-55.

[34] Tang XM, Cheng CH. Borehole Stoneley wave propagation across permeable structures. Geophys Prosp 1993;41:165-87.

[35] Tezuka K, Cheng CH, Tang XM. Modeling of low-frequency Stoneley-wave propagation in an irregular borehole. Geophysics 1997;62: 1047-58.

[36] Bouchon M. A numerical simulation of the acoustic and elastic wavefields radiated by a source in a fluid-filled borehole embedded in a layered medium. Geophysics 1993;58:475-81.

[37] Dong W, Bouchon M, Toksöz MN. Borehole seismic-source radiation in layered isotropic and anisotropic media: boundary element modeling. Geophysics 1995;60:735-47.

[38] Randall CT. Modes of noncircular fluid-filled boreholes in elastic formation. J Acoust Soc Am 1991;89:1002-16.

[39] Tadeu A, Santos P. 3D Wave Propagation in Fluid-filled Irregular Boreholes in Elastic Formations. J Soil Dynam Earthquake Engng 2002 in press. 
[40] Bell JS, Gough DI. Northeast-southwest compressive stress in Alberta - Evidence from oil wells. Earth Planet Sci Lett 1979; $45: 475-82$.

[41] Zheng Z, Kemeny K, Cook NGW. Analysis of borehole breakouts. J Geophys Res 1989;94:171-82.

[42] Bouchon M, Aki K. Discrete wave-number representation of seismic source wavefields. Bull Seismol Soc Am 1977;67:259-77.

[43] Manolis GD, Beskos DE. Boundary element methods in elastodynamics Unwin Hyman. London: Chapman and Hall, 1988.

[44] Beskos DE. Boundary Element Methods in Dynamic Analysis: Part II (1986-1996). Appl Mech Rev 1997;50(3):149-97.

[45] Brebbia CA, Telles JCF, Wrobel LC. Boundary element technique. Berlin: Springer-Verlag, 1984.

[46] Tadeu AJB, Santos PFA, Kausel E. Closed-form integration of singular terms for constant linear and quadratic boundary elements - part I:SH wave propagation. EABE — Engineering Analysis with Boundary Elements 1999;23(8):671-81.

[47] Tadeu AJB, Santos PFA, Kausel E. Closed-form Integration of Singular Terms for Constant Linear and Quadratic Boundary Elements Part II:SV-P Wave Propagation. EABE - Engineering Analysis with Boundary Elements 1999;23(9):757-68.

[48] Pao YH, Mow CC. Diffraction of elastic waves and dynamic stress concentrations. Rand Corporation, 1973.

[49] Kausel E. Frequency domain analysis of undamped systems. J Engng Mech, ASCE 1992;118:121-34.

[50] Ellefsen K.J. Elastic wave propagation along a borehole in an anisotropic medium. PhD, Department of Earth and Planetary Sciences, MIT, 1992. 